\section{PROCESSING OF CALIBRATION MEASUREMENTS ON EZB-3}

\begin{abstract}
The calibration of horizontal circles results in a set of discrete correction values. These corrections are obtained in specific circle positions chosen by the size of the calibration step. For further use it is necessary to know the correction values for any location on a horizontal circle; therefore, it is necessary to know the function of the continuous corrections of a horizontal circle. This could be achieved from the measured values by several methods. In the article two methods are presented for determining this function through the approximation of polynomial and trigonometric series.
\end{abstract}

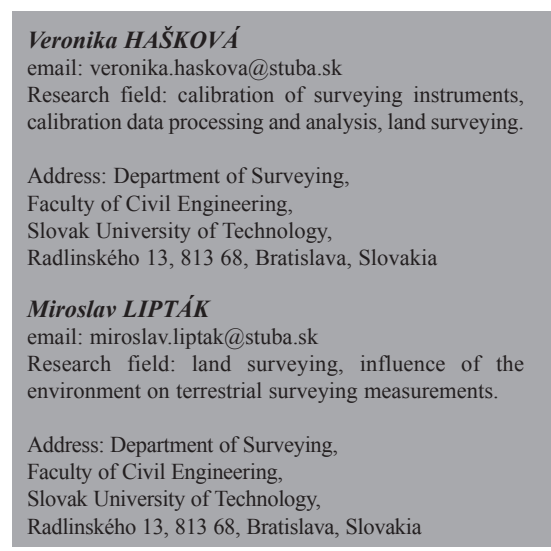

\section{KEY WORDS}

- Calibration,

- correction,

- polynomial series,

- trigonometric series.

\section{INTRODUCTION}

To achieve the required precision of geodetic works in all areas, it is necessary to use such instruments in the measuring process whose properties are in compliance with the specified requirements. One of these features is the consistency, accuracy and repeatability of the values of the horizontal directions. The process for the verification of the quality of theodolite horizontal circles is based on a standard (STN ISO 17123-3); some service centres practise the control of divided circles in accordance with the manufacturer's requirements under the DIN and ISO standards (www.geotech.sk). The EZB-3 calibration equipment of the Slovak Institute of Metrology (SIM) in Bratislava allows for the calibration of horizontal circle surveying instruments using a primary standard of the planar angle of the Slovak Republic.

\section{EZB-3 STANDARD EQUIPMENT}

The EZB-3 standard equipment is used for calibrating a polygonal prism with a combined standard deviation of correction of $u_{c}=0,06 "$.
The equipment was created using a OKT-315 Zeiss Jena rotation dividing table with a servo-drive, where a calibrated polygonal prism is located, as well as two TA- 80 Hilger \& Watts photoelectric autocollimators. Those elements are located on a cast iron base plate with an anti-vibration deposition (Mokroš, 2001). The scheme of the EZB-3 standard equipment is shown in Figure 1. In the base position both autocollimators (AK) are pointed at the neighbouring function surfaces of the traverse; by their stepwise rotation the autocollimators measure the deviations of the normal positions to the traverse function surfaces with regard to the optical axes of the autocollimators.

For calibrating the theodolites a 72-edged polygonal prism (PP) from Starett Webber is located on the position of the calibrated traverse, which is a centric stored calibrated theodolite fitted with a plane mirror. One of the autocollimators, which also serves as a part of the rotation servo-driving (M) of the dividing table, is focused on the polygonal prism. The second autocollimator is situated on the base plate with its optical axis at the height of the theodolite's telescope axis. An example of theodolite calibration on the EZB-3 standard equipment is in Figure 2. 


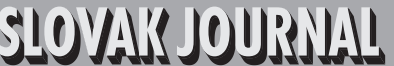

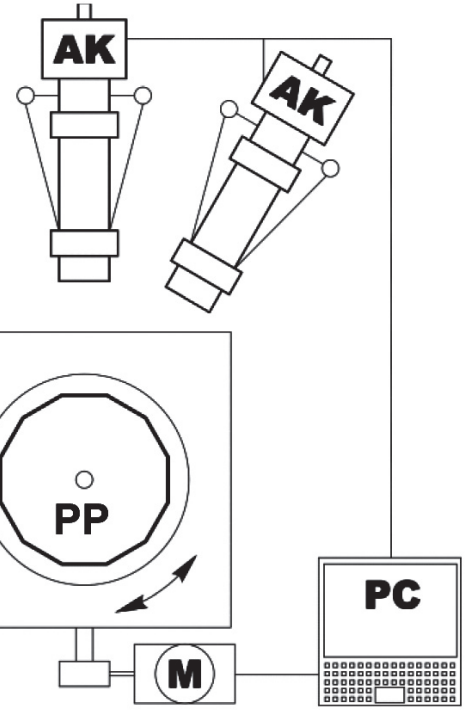

Fig. 1 The scheme of the EZB-3 standard equipment.

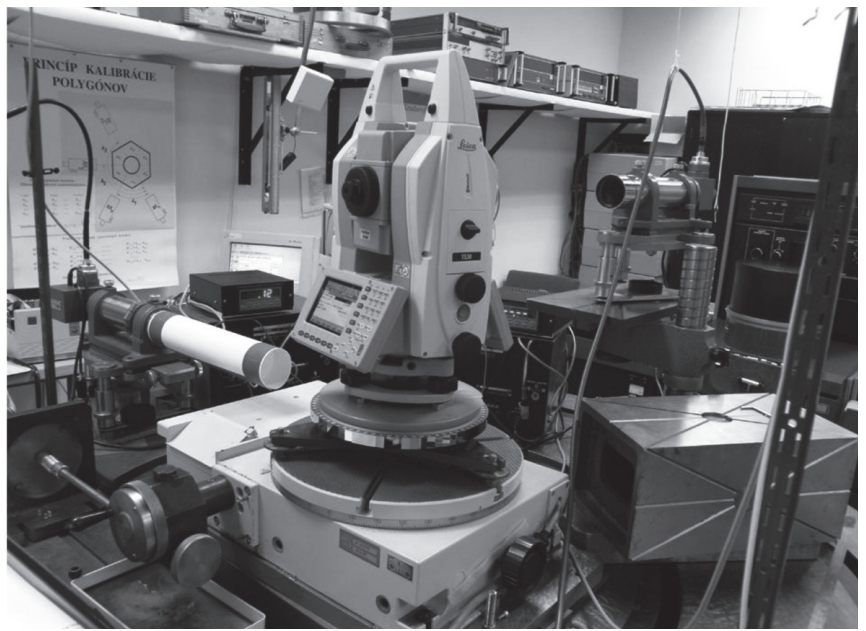

Fig. 2 Leica TS30 calibration on EZB-3.

Before the measurement it is necessary to fulfill the adjustment conditions described in (Mokroš, 2001). After adjustment of the measuring formation, the prism autocollimator is aimed at the required functional surface of the polygonal prism by turning the rotational part of the table with the prism and theodolite. Then the second autocollimator is pointed at the telescope's subsidiary mirror with the help of the theodolite's alidade back rotation. The values of both autocollimators and the value of the theodolite's dividing circle with a given number of repetitions are read. The rotation part of the table rotates to the next prism's functional area, and the process is repeated. One calibration step consists of both autocollimators scanning data into the $\mathrm{PC}$ of the measurement system and the prism and theodolite turning about the required angle (multiple of $5^{\circ}$ ) with a deviation of $0.1 "$.

Provided that the dividing circle of the calibrated theodolite and autocollimator scales is oriented so that by pointing at the first traverse function area, the read value of the theodolite increases and the read value of the autocollimators decreases, then the correction of the theodolite's dividing circle is determined by:

$k_{i}=\frac{360}{N}(j-1)+k_{j}^{P}-B_{i}-a_{j}-b_{i}$

where $k_{i}$ is the correction of the dividing circle of the calibrated theodolite at $i$-point; $N$ is the number of polygonal prism function areas; $k_{j}^{\mathrm{P}}$ is the correction of the function area $j$ of the prism; $B_{i}$ is the value of the theodolite's dividing circle at $i$-point; $a_{j}$ is the reading of the autocollimator scale of the function area $j$ of the polygonal prism; $b_{i}$ is the reading of the autocollimator's scale at the $i$-point of the dividing circle of the calibrated theodolite.

Corrections determined in this way are important for theodolites with the fixed position of the dividing circle. For theodolites, which can change the position of the origin, these corrections provide only an idea about an error course of a horizontal circle's reading system without a possibility of its correction.

\section{CALIBRATION OF A THEODOLITE'S HORIZONTAL CIRCLES ON EZB-3}

Horizontal circles of the Leica TS30 and Leica TCR407 electronic total stations were calibrated on the adapted EZB-3 standard equipment for theodolite calibration. Before the start of the calibration measurement it is necessary to select the calibration cycle counts and calibration steps. The calibration step could be an integral multiple of the nominal angle value between the neighbouring function areas of the polygonal prism. The polygonal prism, which is a part of the EZB-3 equipment, has 72 function areas, so the nominal angle value between the neighbouring function areas is $5^{\circ}$. Our measurements were realised with a $10^{\circ}$ calibration step and 4 calibration cycle counts. Figures 3 and 4 show graphic representations of the horizontal circle corrections from both electronic total station calibrations. For the next processing of the measured data files with $10^{\circ}, 20^{\circ}$ and $30{ }^{\circ}$ calibration steps and cycle counts from 1 to 4 were created. The calculations in the appropriate file were then realised for the average of the measured values. 


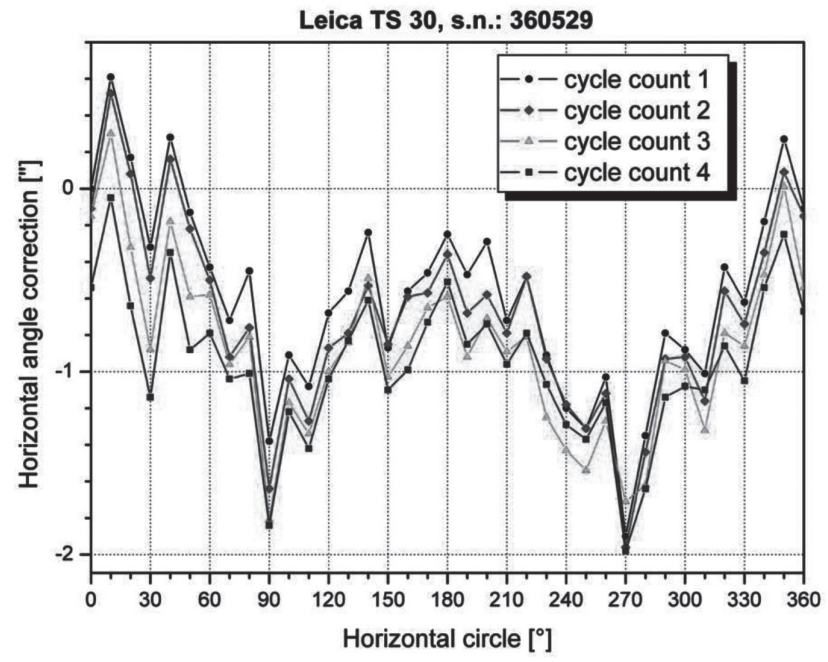

Fig. 3 Graphic representation of the measured horizontal circle correction values of the Leica TS30.

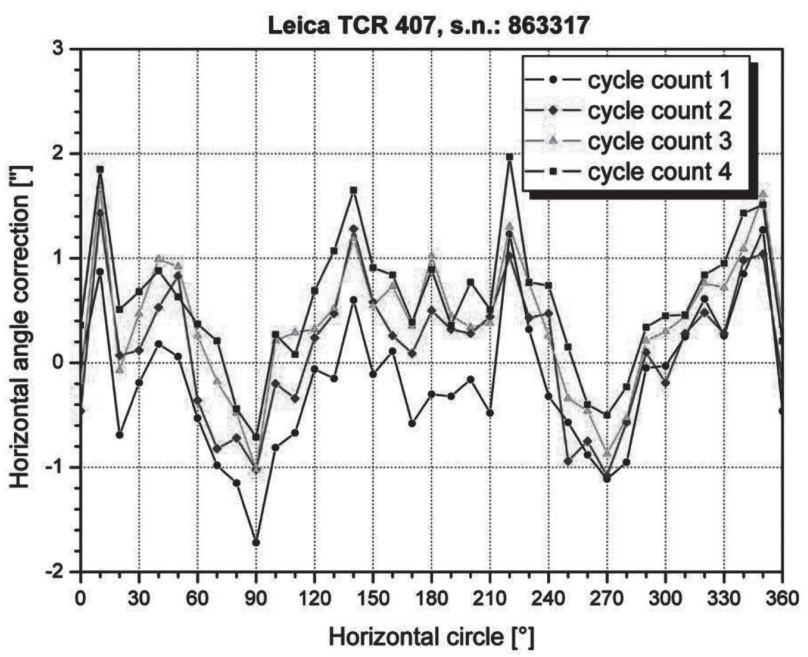

Fig. 4 Graphic representation of the measured horizontal circle correction values of the Leica TCR407.

\section{MEASURED DATA PROCESSING}

The aim of the measured data processing is to find a sufficient function type and its parameter values, which indicate the course of the horizontal circle correction values. There are many options for solving this approximation. In the next part we will focus on an approximation based on a polynomial function and a trigonometric function.
The polynomial function describing the relation between the correction values of a horizontal circle and the changes in the horizontal circle reading, could be written as follows:

$k_{i}=\Theta_{0}+\Theta_{1} \cdot \alpha_{i}+\Theta_{2} \cdot \alpha_{i}^{2}+\ldots+\Theta_{n} \cdot \alpha_{i}^{n}=\sum_{j=0}^{n} \Theta_{j} \cdot \alpha_{i}^{j}$,

where $i=1,2, \ldots, m, k$ is the measurable correction; $\alpha$ is the horizontal circle reading; $\Theta_{j}$ is the unknown polynomial parameters $(j=0,1, \ldots, n)$. The suitable polynomial degree is determined pursuant to various characteristics after estimating the unknown parameters.

Trigonometric functions are used for approximating periodical cases, i.e., cases with a repetitive course at a certain period. The case caused by one source could be described by the simple sinusoid:

$k_{i}=\Theta_{0}+a_{1} \cdot \sin \left(\frac{2 \pi}{P} \cdot \alpha_{i}+\varphi_{1}\right)$

where $i=1,2, \ldots, m, \Theta_{0}$ is a sinusoid drift in a y axis line; $P$ is the period; $a_{i}$ is the sinusoid amplitude; $\varphi_{1}$ is the period's origin shift. Pursuant to the additive relation for the sine of two angles, equation (3) could by modified in the form:

$k_{i}=\Theta_{0}+a_{1} \cdot \sin \left(\frac{2 \pi}{P} \cdot \alpha_{i}\right) \cdot \cos \varphi_{1}+a_{1} \cdot \cos \left(\frac{2 \pi}{P} \cdot \alpha_{i}\right) \cdot \sin \varphi_{1}$.

After implementing the substitution:

$\Theta_{1}=a_{1} \cdot \cos \varphi_{1}, \Theta_{2}=a_{1} \cdot \sin \varphi_{1}$

we can write the equation (4) as follows:

$k_{i}=\Theta_{0}+\Theta_{1} \cdot \sin \left(\frac{2 \pi}{P} \cdot \alpha_{i}\right)+\Theta_{2} \cdot \cos \left(\frac{2 \pi}{P} \cdot \alpha_{i}\right)$,

where $\Theta_{j}$ are unknown parameters $(j=0,1, \ldots, n)$. The original parameters $a_{i}$ and $\varphi_{1}$ can be calculated according to the formulas:

$a_{1}=\sqrt{\Theta_{1}^{2}+\Theta_{2}^{2}}$ and $\varphi_{1}=\operatorname{arctg}\left(\frac{\Theta_{2}}{\Theta_{1}}\right)$.

Each partial periodic error in the divided circle fabrication has a sinusoid course; each trace has a different origin, period and amplitude. After their composition in a closure periodic error, a composite sinusoid arises (Böhm, Radouch, 1978). In horizontal circle corrections only even multiples of the argument are used:

$k_{i}=\Theta_{0}+a_{2} \cdot \sin \left[2 \cdot\left(\frac{2 \pi}{P} \cdot \alpha_{i}\right)+\varphi_{2}\right]+a_{4} \cdot \sin \left[4 \cdot\left(\frac{2 \pi}{P} \cdot \alpha_{i}\right)+\varphi_{4}\right]+\ldots$. 
2011/4 PAGES $18-23$

The formulation of equation (8) as a function of parameters $\Theta_{j}$ $(j=0,1, \ldots, n)$ is an analog to the formulation of the elementary sinusoid. The difference is only more unknown parameters.

The estimation of the unknown parameters of polynomial and trigonometric series is solved by the least squares method. The theoretical model described by equations (2) and (8) can be expressed in a matrix form:

$\mathbf{k}=\mathbf{f}(\boldsymbol{\Theta})$

and after the linearization as follows:

$\mathbf{k}=\mathbf{f}\left(\boldsymbol{\Theta}_{0}\right)+\mathbf{A} \cdot \Delta \boldsymbol{\Theta}$,

where $\boldsymbol{\Theta}$ is a $n$-dimensional vector of unknown parameters; $\boldsymbol{\Theta}_{0}$ is a $n$-dimensional vector with the approximate values of $\boldsymbol{\Theta}$; $\mathbf{A}=\partial \mathbf{f}(\boldsymbol{\Theta}) /\left.\partial \boldsymbol{\Theta}^{\mathrm{T}}\right|_{\Theta=\Theta_{0}}$ is an $m \times n$-dimensional matrix of the first derivates of the vector function $\mathbf{f}(\boldsymbol{\Theta})$ in accordance with $\boldsymbol{\Theta}$, and calculated for the approximate values of the unknown parameters; and $\Delta \boldsymbol{\Theta}$ is a $n$ - dimensional vector of the increments of the unknown parameter $\boldsymbol{\Theta}$. The stochastic model relative to the once realized measurement of each directly measurable parameter could be written as follows:

$\boldsymbol{\xi}=\mathbf{f}\left(\boldsymbol{\Theta}_{0}\right)+\mathbf{A} \cdot \Delta \boldsymbol{\Theta}+\boldsymbol{\varepsilon}, \operatorname{var}(\xi)=\operatorname{var}(\boldsymbol{\varepsilon})=\boldsymbol{\Sigma}$

where $\xi$ is a $m$-dimensional observation vector, whose $i^{\text {th }}$ component models the measurement of the $i^{\text {th }}$ component of the vector $\mathbf{k} ; \boldsymbol{\varepsilon}$ is a $m$-dimensional vector of the random errors; $\boldsymbol{\Sigma}$ is an $m \times m$ - dimensional covariance matrix of vector $\xi$. Because $\boldsymbol{\Theta}$ is estimated in a form $\hat{\boldsymbol{\Theta}}=\mathbf{\Theta}_{0}+\boldsymbol{\Delta} \hat{\boldsymbol{\Theta}}$, the subject of the calculation is the vector of the increments $\Delta \Theta$ in pursuance of realization $\mathbf{x}$ of a random vector $\boldsymbol{\eta}, \boldsymbol{\eta}=\boldsymbol{\xi}-\mathbf{f}\left(\boldsymbol{\Theta}_{0}\right), \operatorname{var}(\boldsymbol{\eta})=\boldsymbol{\Sigma}$. Using the minimizing of a quadratic form for estimating $\boldsymbol{\Delta} \hat{\boldsymbol{\Theta}}$ of the vector of increments $\Delta \boldsymbol{\Delta}$, we get (Kubáčková, 1990):

$\boldsymbol{\Delta} \hat{\boldsymbol{\Theta}}=\left(\mathbf{A}^{\mathrm{T}} \cdot \mathbf{\Sigma}^{-1} \cdot \mathbf{A}\right)^{-1} \cdot \mathbf{A}^{\mathrm{T}} \cdot \mathbf{\Sigma}^{-1} \cdot \mathbf{x}$.

The solution is conditional on the existence of all the necessary matrix inversions that occurred in equation (12).

\section{EVALUATION OF RESULTS}

The calculations necessary for the approximation based on the polynomial function and trigonometric function were realized by Microsoft Excel, PTC MathCAD and Origin Lab Origin software. Both functions were determined for each of the correction value files, which were achieved by the calibration of the horizontal circles of both electronic total stations. The suitable degree of the polynomial function and the type of trigonometric series was realized according to the value of the coefficient of a determination $R^{2}$ and the adjusted coefficient of determination $R_{a d j}^{2}$. Both coefficients indicate the rate of compliance of the empirically measured data with the proposed models. The coefficient of a determination $R^{2}$ value closer to 1 means that the proposed model better represents the empirically measured data. The number of estimated parameters comes only into the estimation of the adjusted coefficient of determination

Tab. 1 Coefficients of determination for Leica TS30.

\begin{tabular}{|c|c|c|c|c|c|c|}
\hline \multirow{2}{*}{ Step/Cycle } & \multicolumn{3}{|c|}{ Polynomial series } & \multicolumn{3}{c|}{ Trigonometric series } \\
\cline { 2 - 7 } & Degree & $\boldsymbol{R}^{\mathbf{2}}$ & $\boldsymbol{R}_{a d j}^{\mathbf{2}}$ & Multiple & $\boldsymbol{R}^{\mathbf{2}}$ & $\boldsymbol{R}_{a d j}^{\mathbf{2}}$ \\
\hline $10^{\circ} / 1$ & $6(0)$ & 0.770 & 0.733 & 2 & 0.549 & 0.523 \\
\hline $10^{\circ} / 2$ & $6(0)$ & 0.778 & $\mathbf{0 . 7 4 3}$ & 2 & 0.576 & 0.563 \\
\hline $10^{\circ} / 3$ & $6(0)$ & 0.762 & 0.723 & 2 & 0.572 & 0.537 \\
\hline $10^{\circ} / 4$ & $6(0)$ & 0.731 & 0.688 & 2 & 0.583 & 0.531 \\
\hline $20^{\circ} / 1$ & $6(0)$ & 0.872 & 0.823 & 2 & 0.626 & $\mathbf{0 . 5 8 0}$ \\
\hline $\mathbf{2 0} / \mathbf{2}$ & $8(0,2)$ & 0.914 & $\mathbf{0 . 8 7 0}$ & 2 & 0.601 & 0.551 \\
\hline $20^{\circ} / 3$ & $8(0,1)$ & 0.904 & 0.856 & 2 & 0.614 & 0.566 \\
\hline $20^{\circ} / 4$ & $8(0,1,2)$ & 0.860 & 0.806 & 2 & 0.661 & 0.593 \\
\hline $30^{\circ} / 1$ & $10(0,1,2,5,6)$ & 0.858 & $\mathbf{0 . 7 5 6}$ & 2 & 0.684 & $\mathbf{0 . 6 2 0}$ \\
\hline $\mathbf{3 0} / \mathbf{2}$ & $10(0,1,2,5,6)$ & 0.839 & 0.724 & 2 & 0.675 & 0.610 \\
\hline $30^{\circ} / 3$ & $10(0,1,2,8,9)$ & 0.788 & 0.363 & 2 & 0.675 & 0.611 \\
\hline $30^{\circ} / 4$ & $10(0,1,2,8,9)$ & 0.725 & 0.529 & 2 & 2 & 2 \\
\hline
\end{tabular}


$R_{a d j}^{2}$. It follows that by the addition of a parameter, which does not increase the model's quality, the value of the coefficient $R^{2}$ is not decreasing; the coefficient $R_{a d j}^{2}$ could decrease and achieve a negative value too.

In Table 1 the values of coefficients $R^{2}$ and $R_{a d j}^{2}$, which were estimated as maximal for the number of calibration steps and cycle counts from the calibration results of the Leica TS30 in a model based on polynomial and trigonometric series, are summarized. Also, the degrees of the polynomial series and the multiples of the argument of the trigonometric series associated with the coefficient $R^{2}$ and $R_{a d j}^{2}$ are given. In the brackets the insignificant parameters are written. These parameters were eliminated from the adjustment results and the remaining parameters were estimated again. For each value of the calibration step the number of cycle counts with a high value of $R_{a d j}^{2}$ is highlighted.

As can be seen from the table, the best results achieved for the Leica TS30 in the case of a polynomial series is by a $20^{\circ}$ calibration step with 2 cycle counts, and the equation, which defines the course of the horizontal circle's correction values, is in the form of a polynomial function of the $8^{\text {th }}$ degree without the parameters $\hat{\Theta}_{0}$ and $\hat{\Theta}_{2}$ :

$$
\begin{aligned}
\hat{k} p_{i} & =1,554 \cdot 10^{-2} \cdot \alpha_{i}-1,826 \cdot 10^{-5} \cdot \alpha_{i}^{3}+3,162 \cdot 10^{-7} \cdot \alpha_{i}^{4}-2,237 \cdot 10^{-9} \cdot \alpha_{i}^{5}+ \\
& +7,859 \cdot 10^{-12} \cdot \alpha_{i}^{6}-1,359 \cdot 10^{-14} \cdot \alpha_{i}^{7}+9,242 \cdot 10^{-18} \cdot \alpha_{i}^{8},
\end{aligned}
$$

in the case of the trigonometric series, the best results achieved by the $30^{\circ}$ calibration step with 2 cycle counts and the equation is here in the form of a trigonometric function with multiples of argument 2 :

$$
\hat{k} t_{i}=-0,81^{\prime \prime}+0,58^{\prime \prime} \cdot \sin \left\{2 \cdot\left(\frac{2 \pi}{P} \cdot \alpha_{i}\right)+84,47^{\circ}\right\} \text {. }
$$

A graphic representation of the curves determined by equations (13) and (14) define the course of the horizontal circle's correction values for the Leica TS30 electronic total station is in Figure 5.

The values of coefficients $R^{2}$ and $R_{a d j}^{2}$, which were estimated from the calibration results of the Leica TCR407 electronic total station

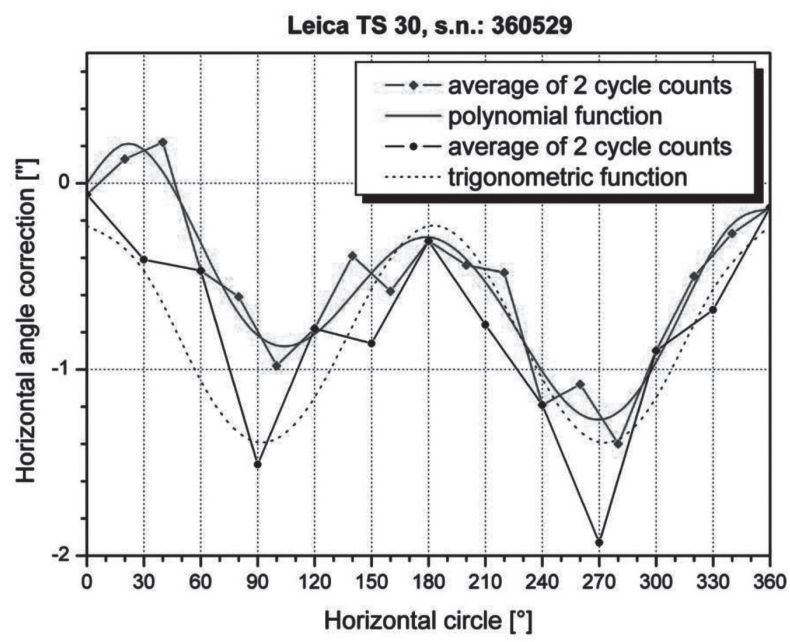

Fig. 5 Curves determining the corrections course of the horizontal circle for the Leica TS30.

Tab. 2 Coefficients of determination for Leica TCR407.

\begin{tabular}{|c|c|c|c|c|c|c|}
\hline \multirow{2}{*}{ Step/Cycle } & \multicolumn{3}{|c|}{ Polynomial series } & \multicolumn{3}{c|}{ Trigonometric series } \\
\cline { 2 - 7 } & Degree & $\boldsymbol{R}^{\mathbf{2}}$ & $\boldsymbol{R}_{a d j}^{\mathbf{2}}$ & Multiple & $\boldsymbol{R}^{\mathbf{2}}$ & $\boldsymbol{R}_{a d j}^{\mathbf{2}}$ \\
\hline $10^{\circ} / 1$ & $6(0)$ & 0.464 & 0.377 & 2,4 & 0.555 & 0.499 \\
\hline $10^{\circ} / 2$ & $6(0)$ & 0.490 & 0.408 & 2,4 & 0.629 & 0.595 \\
\hline $10^{\circ} / 3$ & $6(0)$ & 0.499 & $\mathbf{0 . 4 1 8}$ & $2,4,6$ & 0.642 & 0.584 \\
\hline $10^{\circ} / 4$ & $6(0)$ & 0.493 & 0.412 & 2,4 & 0.631 & 0.585 \\
\hline $20^{\circ} / 1$ & $10(0)$ & 0.777 & 0.554 & 2,4 & 0.687 & 0.624 \\
\hline $20^{\circ} / 2$ & $10(0)$ & 0.811 & 0.622 & $2,4,6$ & 0.827 & $\mathbf{0 . 7 6 1}$ \\
\hline $20^{\circ} / 3$ & $10(0)$ & 0.815 & $\mathbf{0 . 6 3 0}$ & $2,4,6$ & 0.828 & 0.742 \\
\hline $20^{\circ} / 4$ & $10(0)$ & 0.803 & 0.606 & $2,4,6$ & 0.817 & 0.725 \\
\hline $\mathbf{3 0} / \mathbf{1}$ & $10(0)$ & 0.956 & $\mathbf{0 . 8 2 4}$ & 2,4 & 0.771 & 0.656 \\
\hline $\mathbf{3 0} / \mathbf{2}$ & $10(0)$ & 0.950 & 0.800 & 2,4 & 0.849 & $\mathbf{0 . 7 7 3}$ \\
\hline $30^{\circ} / 3$ & $10(0)$ & 0.935 & 0.741 & 2,4 & 0.812 & 0.749 \\
\hline $30^{\circ} / 4$ & $10(0)$ & 0.945 & 0.780 & 2,4 & 0.829 & 0.771 \\
\hline
\end{tabular}


in the model based on the polynomial and trigonometric series, are presented in Table 2 . The number of cycle counts with a high value of $R_{a d j}^{2}$ for each value of the calibration step is highlighted.

From the results we can see that for Leica TCR407 the best results achieved in the case of a polynomial series is by the $30^{\circ}$ calibration step with a 1 cycle count, and the equation, which defines the course of the horizontal circle's correction values, is in the form of a polynomial function of the $10^{\text {th }}$ degree without parameter $\hat{\Theta}_{0}$ :

$$
\begin{aligned}
\hat{k} p_{i} & =-0,823 \cdot \alpha_{i}+6,811 \cdot 10^{-2} \cdot \alpha_{i}^{2}-2,188 \cdot 10^{-3} \cdot \alpha_{i}^{3}+3,646 \cdot 10^{-5} \cdot \alpha_{i}^{4}- \\
& -3,561 \cdot 10^{-7} \cdot \alpha_{i}^{5}+2,151 \cdot 10^{-9} \cdot \alpha_{i}^{6}-8,142 \cdot 10^{-12} \cdot \alpha_{i}^{7}+1,881 \cdot 10^{-14} \cdot \alpha_{i}^{8}- \\
& -2,426 \cdot 10^{-17} \cdot \alpha_{i}^{9}+1,337 \cdot 10^{-20} \cdot \alpha_{i}^{10}
\end{aligned}
$$

in the case of trigonometric series the best results achieved by the $30^{\circ}$ calibration step with 2 cycle counts and the equation is in the form of a trigonometric function with multiples of argument 2 and 4 :

$$
\begin{aligned}
\hat{k} t_{i}= & -0,22^{\prime \prime}+0,46^{\prime \prime} \cdot \sin \left\{2 \cdot\left(\frac{2 \pi}{P} \cdot \alpha_{i}\right)+106,63^{\circ}\right\}+ \\
& +0,45^{\prime \prime} \cdot \sin \left\{4 \cdot\left(\frac{2 \pi}{P} \cdot \alpha_{i}\right)+266,00^{\circ}\right\} .
\end{aligned}
$$

A graphic representation of the curves determined by equations (15) and (16), which define the course of the horizontal circle's correction values for the Leica TCR407 electronic total station, is in Figure 6.

\section{CONCLUSION}

In the paper the procedure for calibrating the horizontal circle of the Leica TS30 and Leica TCR407 electronic total stations on the EZB-3 standard equipment of the Slovak Institute of Metrology in Bratislava was described. For both instruments the suitable function,

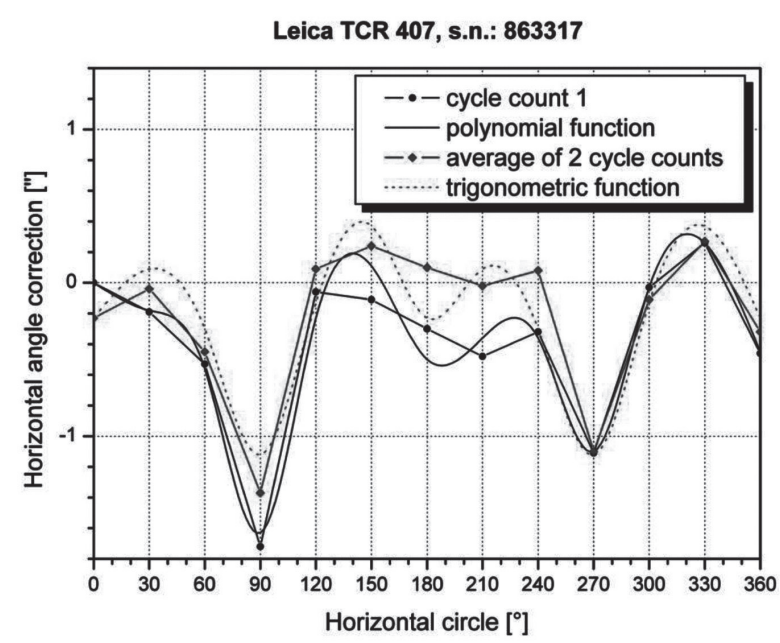

Fig. 6 Curves determining the corrections course of the horizontal circle for the Leica TCR407.

which determines the course of the horizontal circle's correction values depending on the value of the horizontal circle reading, was determined. The function approximation was based on the polynomial function and trigonometric function. According to the value of the coefficients of the determination, the suitable degree of the polynomial function and the type of the trigonometric series for both electronic total stations was selected. From the estimated results it can be said that for determining the course of the horizontal circle's correction values, the calibration of both instruments with a $30^{\circ}$ or $20^{\circ}$ calibration step up to 2 calibration cycle counts is sufficient.

\section{ACKNOWLEDGEMENT}

The authors are grateful to the Slovak Grant Agency VEGA for its financial support of this project. The project registration number is 1/0142/10.

\section{REFERENCES}

[1] Böhm, J., Radouch, V.: Vyrovnávací počet. Adjustment computation. Prague: Kartografie, 1978. 510 pp. (in Czech).

[2] <http://www.geotech.sk/Sluzby/Kalibracie.html>.

[3] Kubáčková, L.: Metódy spracovania experimentálnych údajov. Methods of Experimental Data Processing. Bratislava: VEDA, 1990. 324 pp. ISBN 80-224-0104-8 (in Slovak).

[4] Mokroš, J. Kalibrácia horizontálneho kruhu teodolitov.
Calibration of theodolite horizontal circle. In: Metrológia v geodézii. Bratislava: Katedra geodetických základov SvF, 2001. ISBN 80-227-1617-0, pp. 123-130 (in Slovak).

[5] STN ISO 17123-3. Optics and optical Instruments - Field procedures for testing geodetic and surveying instruments - Part 3: Theodolites. 2001. 